\title{
Percepción y conocimientos de los profesionales sanitarios de una unidad de Nefrología sobre la higiene de manos: estudio comparativo
}

\author{
José Luis Cobo Sánchez*, Raquel Pelayo Alonso*, Raquel Menezo Viadero*, Elena Incera Setién*, Magdalena \\ Gándara Revuelta*, Luis Mariano López López ${ }^{* *}$ \\ Diplomados en Enfermería. * Servicio de Nefrología, ${ }^{*}$ Servicio de Traumatología. Hospital Universitario Marqués de \\ Valdecilla. Santander
}

\section{Resumen \\ Un conocimiento adecuado sobre la higiene de las ma- nos por parte de los profesionales sanitarios, es el pri- mer paso para la adherencia a cualquier programa de disminución de infección relacionada con la asistencia sanitaria.}

\section{Objetivo}

Evaluar el nivel de conocimientos y percepciones respecto a la higiene de manos entre los profesionales sanitarios de nuestra unidad de Nefrología, y compararlos con los de otra unidad de nuestro centro (Traumatología).

\section{Material y método}

Estudio prospectivo descriptivo comparativo en 69 profesionales sanitarios. Para evaluar los conocimientos y percepciones respecto a la higiene de manos se utilizó un cuestionario de 26 ítems basado en cuestionarios desarrollados por la Organización Mundial de la Salud.

\section{Resultados}

$73 \%$ utiliza regularmente preparados de base alcohólica para higiene de manos (Traumatología 96,9\%, $p=0,007$ ). $94 \%$ piensa que las manos de los profesionales sanitarios cuando no están limpias son la principal vía de transmisión de gérmenes patógenos (Traumatología: $87,1 \%, p=N S$ ). $75,7 \%$ piensan que el entorno/superficies del hospital es la principal fuente de gérmenes patógenos (Traumatología: $68,8 \%$ ) y $16 \%$ que son los microrganismos ya presentes en el paciente (Traumatología: 25\%) ( $p=N S$ ). Percepciones: $89 \%$ consideran alta la repercusión de las infecciones re-

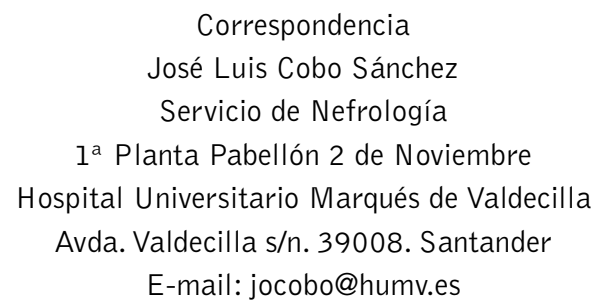

lacionadas con la asistencia sanitaria en el desenlace de la enfermedad (Traumatología: 59,4\%, $p=0,009$ ). Respecto a las medidas más efectivas para mejorar la higiene de manos destacan la formación de los profesionales sanitarios, recibir retroalimentación regularmente sobre cómo realizar la higiene de manos y que exista un preparado de base alcohólica en cada punto de atención.

\section{Conclusiones}

Los profesionales sanitarios de Nefrología, al igual que los de Traumatología, en general tienen conocimientos y percepciones adecuados sobre la higiene de manos.

PALABRAS CLAVE:

- HIGIENE DE LAS MANOS

- PROFESIONAL SANITARIO

- CONOCIMIENTOS, ACTITUDES Y PRÁCTICA EN SALUD

- DIÁLISIS RENAL

- TRAUMATOLOGÍA

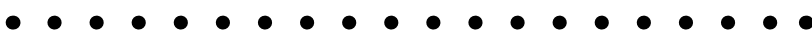
Hand hygiene perceptions and knowledge of healthcare workers in a Nephrology Unit: a comparative study.

\section{Abstract}

Adequate knowledge about hand hygiene ( $\mathrm{Hh}$ ) by healthcare workers (HCW), is the first step for adhering to any decreased infection related with healthcare (IRHC) program.

Aim

To evaluate $\mathrm{Hh}$ perceptions and knowledge of HCW in a Nephrology Unit and compare it with another unit in our hospital (Trauma).

\section{Methodology}

Comparative prospective study in $69 \mathrm{HCW}$. A 26-item questionnaire, based on questionnaires developed by WHO to assess knowledge and perceptions of $\mathrm{Hh}$ in HCW was used. 


\section{Results}

$73 \%$ regularly use alcohol-based preparations for $\mathrm{Hh}$ (Trauma 96.9\%, $p=0.007$ ). 94\% think that the hands of the HCW when they are not clean are the main route of transmission of pathogens (Trauma: $87.1 \%, p=N S$ ). $75.7 \%$ think that the environment / hospital surfaces is the main source of pathogens (Trauma: $68.8 \%$ ) and $16 \%$ which are the microorganisms already present in the patient (Trauma: $25 \%)(p=N S$ ). Perceptions: $89 \%$ consider the impact of high IRHC in the outcome of the disease (Trauma: 59.4\%, $p=0.009$ ). Regarding the most effective measures to improve the $\mathrm{Hh}$ include the formation of $\mathrm{HCW}$, receive regular feedback on how to make the $\mathrm{Hh}$ and that a alcohol-based handrub is present at each point of care.

\section{Conclusions}

All healthcare workers have adequate knowledge and perceptions about $\mathrm{Hh}$.

\section{KEY WORDS:}

- HAND HYGIENE

- HEALTHCARE PROFESSIONALS

- HEALTH KNOWLEDGE, ATTITUDES, PRACTICE

- RENAL DIALYSIS

- TRAUMATOLOGY

\section{Introducción}

La prevalencia de infecciones nosocomiales en hospitales españoles en el año 2011 fue del 6,3\%1, lo que constituye un grave problema de seguridad del paciente ya que afectan a miles de personas, complican los cuidados de los pacientes, contribuyen a su muerte 0 a su discapacidad, favorecen la aparición de resistencia a los antibióticos y generan un considerable coste adicional a la enfermedad².

En España, la infección es la segunda causa de muerte entre los pacientes con enfermedad renal, tras la patología cardiovascular ${ }^{3}$. Los pacientes con enfermedad renal, en especial los pacientes en diálisis, presentan un riesgo mayor de padecer una infección relacionada con la asistencia sanitaria (IRAS) debido al estado de inmunodepresión y a la frecuente exposición a bacterias nosocomiales y procedimientos invasivos ${ }^{4}$. La higiene de las manos (HM) es la medida más sencilla y eficaz para prevenir la transmisión de microorganismos a través del contacto, sin embargo, números estudios han puesto de manifiesto su bajo grado de cumplimiento entre los profesionales sanitarios (PS $)^{5-8}$. Un conocimiento adecuado sobre la HM por parte de los
PS, es el primer paso para la adherencia a cualquier programa de disminución de IRAS. Los programas de formación e información continuos sobre la HM, junto con la introducción de soluciones hidroalcohólicas para su realización, han demostrado ser las medidas más eficaces para mejorar la adherencia a la $\mathrm{HM}^{9,10}$. En nuestro hospital, desde hace 3 años se está llevando a cabo un programa integral para la vigilancia, prevención y control de las IRAS, que incluye la información y formación del personal sobre la importancia de las IRAS, haciendo especial hincapié en la HM. Por este motivo, nos propusimos evaluar el nivel de conocimientos y percepciones respecto a la HM entre los PS de nuestra unidad de Nefrología, y compararlos con los de otra unidad de nuestro centro.

\section{Material y método}

Estudio prospectivo descriptivo comparativo en 69 PS: 37 de Nefrología y 32 de Traumatología. Se eligió el servicio de Traumatología por ser una unidad con pacientes susceptibles de sufrir IRAS al igual que los de Nefrología debido al alto número de procedimientos invasivos a los que se ven sometidos, y por ser una unidad de hospitalización en la que se habían llevado a cabo las mismas estrategias dentro de la campaña de prevención de infecciones hospitalarias en nuestro centro. Para evaluar los conocimientos y percepciones respecto a la $\mathrm{HM}$ se utilizó un cuestionario de 26 ítems basado en los cuestionarios desarrollados por la Organización Mundial de la Salud (OMS) a este respecto ${ }^{11,12}$.

- 5 preguntas sobre datos sociodemográficos: sexo, edad, profesión, años de experiencia profesional y unidad clínica.

- 10 preguntas sobre conocimientos respecto a la HM: preguntadas cerradas de respuesta múltiple.

- 11 preguntas sobre percepciones: 3 preguntas con una escala tipo Likert de 4 puntos (de "muy baja" a "muy alta"), 5 preguntas con una escala tipo Likert de 0 a 6 puntos (siendo 0 nada efectivo/ ninguna importancia/ ningún esfuerzo, y 6 muy efectivo/ mucha importancia/ mucho esfuerzo); y 3 preguntas abiertas sobre distintos porcentajes acerca de creencias.

El tiempo necesario para cumplimentar los cuestionarios osciló entre 20 y 30 minutos. Los cuestionarios fueron entregados por miembros del equipo investigador a los PS de ambas unidades (muestreo incidental) y se contestaron de forma anónima. Las instrucciones de los encuestadores se limitaron a una presentación formal, 
instrucciones de dónde depositarlo y el agradecimiento personal, sin responder preguntas.

Los datos recogidos fueron analizados mediante el paquete estadístico SPSS versión 15.0 para Windows. Se realizó un análisis descriptivo de los resultados obtenidos: para las variables categóricas se calcularon los porcentajes y para las variables continuas se calculó la media. Para la inferencia estadística se utilizaron los tests de Chi cuadrado y de la T de Student para muestras independientes. Los resultados se consideraron significativos si el nivel crítico observado era inferior al $5 \%(p<0,05)$.

\section{Resultados}

Las características sociodemográficas del PS de Nefrología fueron: $89,2 \%$ mujeres, la mayoría enfermeras (62,2\% enfermeras, $29,7 \%$ auxiliares de enfermería, $5,4 \%$ nefrólogos), con una edad media de 43 años (rango 20-64 años, desviación típica 10,19 años) y una experiencia profesional mayoritaria de 11 a 20 años ( $<5$ años 8,1\%, 5-10 años 24,3\%, $11-20$ años $29,7 \%$, $21-25$ años 18,9\%, >25 años 18,9\%). Las características sociodemográficas del PS de Traumatología fueron: $78,1 \%$ mujeres, mayoritariamente enfermeras (37,5\% enfermeras, $31,3 \%$ auxiliares de enfermería, $31,3 \%$ médico), con una edad media de 39,9 años (rango 25-60 años, desviación típica 9,28 años) y una experiencia profesional mayoritaria de 11 a 20 años $(<5$ años $12,5 \%, 5-10$ años $21,9 \%$, $11-20$ años 34,4\%, 21 25 años $21,9 \%$, >25 años $9,4 \%$ ).

El $81,1 \%$ de los PS de Nefrología refería haber recibido información reglada sobre $\mathrm{HM}$ en los últimos 3 años $(82,6 \%$ de las enfermeras, $90,9 \%$ de las auxiliares y $50 \%$ de los médicos) frente al $65,6 \%$ de los PS de Traumatología $(66,7 \%$ de las enfermeras, $70 \%$ de las auxiliares y $60 \%$ de los médicos) ( $p=N S$ ). Entre los PS de Nefrología el $73 \%$ refería utilizar un preparado de base alcohólica para la HM $(69,6 \%$ de las enfermeras, $81,8 \%$ de las auxiliares, $100 \%$ de los médicos), frente a aun $96,9 \%$ de los PS de Traumatología ( $100 \%$ de las enfermeras, $100 \%$ de las auxiliares, $90 \%$ de los médicos) $(p=0,007)$.
Los resultados sobre conocimientos acerca de la $\mathrm{HM}$ se muestran en la Tabla 1. Respecto a qué tipo de HM se requiere ante distintas situaciones, los PS respondieron: antes de la palpación abdominal (PS Nefrología: fricción $70,3 \%$, lavado $21,6 \%$, ninguno $8,1 \%$; PS Traumatología: fricción $74,2 \%$, lavado $12,9 \%$, ninguno 9,7\%, lavado y fricción 3,2\%; $p=N S$ ), antes de poner una inyección (PS Nefrología: fricción $24,3 \%$, lavado $62,2 \%$, ninguno $5,4 \%$, lavado y fricción $8,1 \%$; PS Traumatología: fricción $67,7 \%$, lavado $19,4 \%$, ninguno 9,7\%, lavado y fricción 3,2\%; $p=0,001$ ), después de vaciar una cuña (PS Nefrología: fricción 2,7\%, lavado 89,2\%, lavado y fricción 8,1\%; PS Traumatología: fricción 16,1\%, lavado $77,4 \%$, lavado y fricción $6,5 \% ; p=N S)$, después de quitarse los guantes (PS Nefrología: fricción 2,7\%, lavado 89,2\%, lavado y fricción $8,1 \%$; PS Traumatología: fricción $71 \%$, lavado $16,1 \%$, ninguno 6,5\%, lavado y fricción $6,5 \% ; p<0,001)$, después de hacer la cama del paciente (PS Nefrología: fricción 45,9\%, lavado 51,4\%, ninguno 2,7\%; PS Traumatología: fricción 74,2\%, lavado $12,9 \%$, lavado y fricción $12,9 \% ; p=0,002)$, tras la exposición visible a sangre (PS Nefrología: fricción 8,1\%, lavado 64,9\%, lavado y fricción 27\%; PS Traumatología: fricción $12,9 \%$, lavado $77,4 \%$, ninguno $3,2 \%$, lavado y fricción $6,5 \% ; p=N S$ ). El $56,8 \%$ de los PS de Nefrología señaló los 5 momentos recomendados por la OMS para realizar la HM frente a un $65,6 \%$ de los PS de Traumatología ( $p=N S$ ). En ambos grupos se consideró el uso de joyas y de uñas postizas así cómo la presencia de lesiones cutáneas como circunstancias que han de evitarse ya que se asocian con una mayor probabilidad de colonización de las manos por microorganismos patógenos, y que el uso regular de cremas no se asocia con esta probabilidad.

Los resultados acerca de percepciones sobre la $\mathrm{HM}$ se muestran en la Tabla 2. El 89,18\% de los PS de Nefrología frente a un 59, 37\% de los PS de Traumatología consideraban alta la repercusión que tienen las IRAS en el desenlace de la enfermedad del paciente $(p=0,009)$. Los PS de ambos grupos consideraban como muy alta la eficacia de la HM en la prevención de las IRAS (PS Nefrología 59,4\% vs PS Traumatología 56,25\%; $p=N S$ ). En ambos grupos se consideraba que se daba una prioridad alta o muy alta a la HM en nuestro hospital (PS Nefrología $90,62 \%$ vs PS Traumatología $87,5 \% ; p=N S$ ). 
Tabla 1. Resultados preguntas sobre conocimientos (HM: higiene de las manos, FC: fluidos corporales, FM: fricción de las manos, PBA: preparados de base alcohólica, $\left.{ }^{*}: p<0,05\right)$.

¿Cuál de las siguientes es la principal vía de transmisión cruzada de microorganismos potencialmente patógenos entre los pacientes en los centros sanitarios?

¿Cuál es la fuente más frecuente de gérmenes causantes de infecciones relacionadas con la atención sanitaria?

¿Cuál de las siguientes acciones de HM previene la transmisión de microorganismos al paciente?

¿Cuál de las siguientes acciones de HM previene la transmisión de microorganismos al profesional sanitario?

¿Cuáles de las siguientes afirmaciones sobre la FM con PBA y el LM con agua y jabón son verdaderas?

¿Cuál es el tiempo mínimo necesario para que FM con PBA elimine los gérmenes de las manos?
Las manos de los profesionales sanitarios cuando no están limpias.

El aire que circula en el hospital

La exposición de los pacientes a superficies colonizadas por gérmenes

Compartir objetos no invasivos entre los pacientes

El sistema de agua del hospital

El aire del hospital

Microorganismos ya presen-

tes en el paciente

El entorno (las superficies) del hospital

Antes de tocar al paciente

Inmediatamente después del riesgo de exposición a FC

Después del contacto con

el entorno inmediato del

paciente

Inmediatamente antes de un procedimiento limpio / aséptico

Después de tocar al paciente

Inmediatamente después del riesgo de exposición a $\mathrm{FC}$

Inmediatamente antes de un procedimiento limpio / aséptico

Después del contacto con el entorno inmediato del paciente

La fricción es más rápida que el LM

La fricción causa más sequedad de la piel que el LM

La fricción es más eficaz contra los gérmenes que el LM

Se recomienda realizar el LM y la FM de forma secuencial

20 segundos

3 segundos

Iminuto

10 segundos

\begin{tabular}{|c|c|c|c|c|c|c|c|}
\hline \multicolumn{4}{|c|}{ NEFROLOGIA } & \multicolumn{4}{c|}{ TRAUMATOLOGIA } \\
\hline Total & $\begin{array}{c}\text { Enfer- } \\
\text { mera }\end{array}$ & Auxiliar & Médico & Total & $\begin{array}{c}\text { Enfer- } \\
\text { mera }\end{array}$ & Auxiliar & Médico \\
\hline $94,6 \%$ & $95,7 \%$ & $90,9 \%$ & $100 \%$ & $87,1 \%$ & $90,9 \%$ & $90 \%$ & $80 \%$ \\
\hline- & - & - & - & $3,2 \%$ & $9,1 \%$ & - & - \\
\hline- & - & - & - & $6,5 \%$ & - & - & $20 \%$ \\
\hline $5,4 \%$ & $4,3 \%$ & $9,1 \%$ & - & $3,2 \%$ & - & $10 \%$ & - \\
\hline
\end{tabular}

\begin{tabular}{|c|}
\hline $2,7 \%$ \\
\hline
\end{tabular}

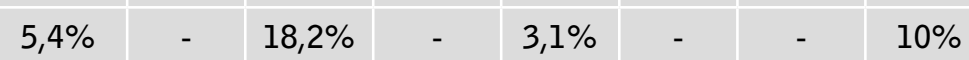

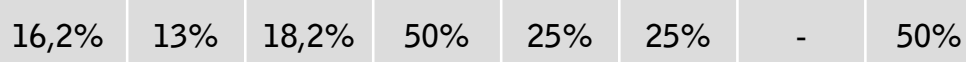

\begin{tabular}{|l|l|l|l|l|l|l|l|}
\hline $75,5 \%$ & $87 \%$ & $54,5 \%$ & $50 \%$ & $68,8 \%$ & $75 \%$ & $90 \%$ & $40 \%$
\end{tabular}

\begin{tabular}{|l|l|l|l|l|l|l|l|}
\hline $89,2 \%$ & $87 \%$ & $90,9 \%$ & $100 \%$ & $87,5 \%$ & $91,7 \%$ & $80 \%$ & $90 \%$ \\
\hline $70,3 \%$ & $78,3 \%$ & $54,5 \%$ & $50 \%$ & $71,9 \%$ & $83,3 \%$ & $60 \%$ & $70 \%$ \\
\hline $64,9 \%$ & $69,6 \%$ & $45,5 \%$ & $100 \%$ & $75 \%$ & $100 \%$ & $60 \%$ & $60 \%$ \\
\hline $86,5 \%$ & $95,7 \%$ & $63,6 \%$ & $100 \%$ & $78,1 \%$ & $91,7 \%$ & $60 \%$ & $80 \%$ \\
\hline
\end{tabular}

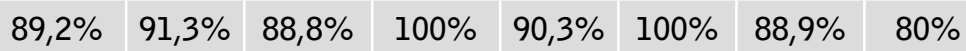

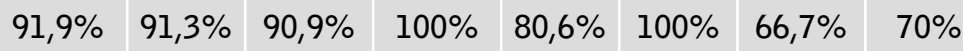

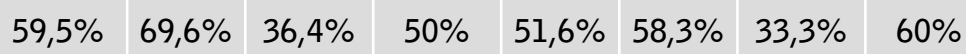

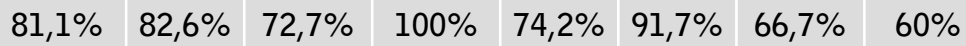

\begin{tabular}{|c|c|c|c|c|c|c|c|}
\hline $73 \%$ & $69,6 \%$ & $72,7 \%$ & $100 \%$ & $74,2 \%$ & $83,3 \%$ & $77,8 \%$ & $60 \%$ \\
\hline $78,4 \%$ & $78,3 \%$ & $72,7 \%$ & $100 \%$ & $58,1 \%$ & $50 \%$ & $55,6 \%$ & $70 \%$ \\
\hline $21,6 \% *$ & $21,7 \%$ & $9,1 \%$ & $50 \%$ & $51,6 \%$ & $75 \%$ & $33,3 \%$ & $40 \%$ \\
\hline $62,2 \%$ & $60,9 \%$ & $63,3 \%$ & $100 \%$ & $74,2 \%$ & $75 \%$ & $77.8 \%$ & $70 \%$ \\
\hline $64,9 \%$ & $69,6 \%$ & $54,5 \%$ & $100 \%$ & $50 \%$ & $66,7 \%$ & $20 \%$ & $60 \%$ \\
\hline $5,4 \%$ & $4,3 \%$ & $9,1 \%$ & - & $6,3 \%$ & $8,3 \%$ & $10 \%$ & - \\
\hline $18,9 \%$ & $13 \%$ & $27,3 \%$ & - & $28,1 \%$ & $8,3 \%$ & $60 \%$ & $20 \%$ \\
\hline $10,8 \%$ & $13 \%$ & $9,1 \%$ & - & $15,6 \%$ & $16,7 \%$ & $10 \%$ & $20 \%$ \\
\hline
\end{tabular}


[ José Luis Cobo Sánchez, et al ]

Percepción y conocimientos de los profesionales sanitarios de una unidad de Nefrología sobre la higiene de manos: estudio comparativo

Tabla 2.- Resultados preguntas sobre percepciones (HM: higiene de las manos, PBA: preparado de base alcohólica, *:p<0,05).

\begin{tabular}{|c|c|c|c|c|c|c|c|c|c|}
\hline & \multicolumn{4}{|c|}{ NEFROLOGIA } & \multicolumn{4}{|c|}{ TRAUMATOLOGIA } \\
\hline & & Total & $\begin{array}{l}\text { Enfer- } \\
\text { mera }\end{array}$ & Auxiliar & Médico & Total & $\begin{array}{l}\text { Enfer- } \\
\text { mera }\end{array}$ & Auxiliar & Médico \\
\hline \multicolumn{2}{|c|}{$\begin{array}{l}\text { En su opinión, ¿cuál es el porcentaje medio } \\
\text { de pacientes hospitalizados que contraen } \\
\text { una infección relacionada con la atención } \\
\text { sanitaria? }\end{array}$} & $41,13 \%$ & $39,2 \%$ & $43,33 \%$ & $30 \%$ & $35,8 \%$ & $21,6 \%$ & $48 \%$ & $31,25 \%$ \\
\hline \multicolumn{2}{|c|}{$\begin{array}{l}\text { De media, ¿en qué porcentaje de situaciones } \\
\text { en las que se requiere, realmente realizan } \\
\text { la HM los profesionales sanitarios de } \\
\text { su hospital, ya sea mediante un PBA o } \\
\text { lavándoselas? }\end{array}$} & $70,71 \%$ & $71,79 \%$ & $74 \%$ & $60 \%$ & $54,15 \%$ & $70 \%$ & $73 \%$ & $22,6 \%$ \\
\hline \multicolumn{2}{|c|}{$\begin{array}{l}\text { De media, ¿en qué porcentaje de situaciones } \\
\text { en las que se requiere, realmente realiza } \\
\text { usted la HM ya sea con PBA o lavándoselas? }\end{array}$} & $81,91 \%$ & $83,82 \%$ & $84,09 \%$ & $40 \%$ & $84,31 \%$ & $80,5 \%$ & $90,5 \%$ & $82,67 \%$ \\
\hline \multirow{8}{*}{$\begin{array}{l}\text { ¿Cuál } \\
\text { sería la } \\
\text { efectividad } \\
\text { de las } \\
\text { siguientes } \\
\text { medidas } \\
\text { destinadas } \\
\text { a mejorar } \\
\text { de forma } \\
\text { permanente } \\
\text { la higiene } \\
\text { de las } \\
\text { manos en su } \\
\text { centro? }\end{array}$} & $\begin{array}{l}\text { Que los directivos de su } \\
\text { institución apoyen y promuevan } \\
\text { abiertamente la HM. }\end{array}$ & $\begin{array}{c}4,64 \\
\text { puntos }\end{array}$ & $\begin{array}{c}4,57 \\
\text { puntos }\end{array}$ & $\begin{array}{c}4,64 \\
\text { puntos }\end{array}$ & $\begin{array}{c}5,5 \\
\text { puntos }\end{array}$ & $\begin{array}{c}4,43 \\
\text { puntos }\end{array}$ & $\begin{array}{c}3,67 \\
\text { puntos }\end{array}$ & $\begin{array}{c}5,8 \\
\text { puntos }\end{array}$ & 4 puntos \\
\hline & $\begin{array}{l}\text { Que en su centro exista } \\
\text { un PBA en cada punto de } \\
\text { atención. }\end{array}$ & $\begin{array}{c}5,08 \\
\text { puntos }\end{array}$ & $\begin{array}{l}5,09 \\
\text { puntos }\end{array}$ & 5 puntos & $\begin{array}{c}5 \\
\text { puntos }\end{array}$ & $\begin{array}{c}5,31 \\
\text { puntos }\end{array}$ & $\begin{array}{c}4,92 \\
\text { puntos }\end{array}$ & $\begin{array}{c}5,9 \\
\text { puntos }\end{array}$ & $\begin{array}{c}5,2 \\
\text { puntos }\end{array}$ \\
\hline & $\begin{array}{l}\text { Que existan carteles sobre HM } \\
\text { en los puntos de atención a } \\
\text { modo de recordatorio. }\end{array}$ & $\begin{array}{c}4,05 \\
\text { puntos }\end{array}$ & $\begin{array}{c}4,17 \\
\text { puntos }\end{array}$ & $\begin{array}{c}3,64 \\
\text { puntos }\end{array}$ & $\begin{array}{c}5,5 \\
\text { puntos }\end{array}$ & $\begin{array}{c}4,43 \\
\text { puntos }\end{array}$ & $\begin{array}{c}3,67 \\
\text { puntos }\end{array}$ & $\begin{array}{c}5,5 \\
\text { puntos }\end{array}$ & $\begin{array}{c}4,3 \\
\text { puntos }\end{array}$ \\
\hline & $\begin{array}{l}\text { Que todos los profesionales } \\
\text { sanitarios reciban formación } \\
\text { sobre la HM. }\end{array}$ & $\begin{array}{c}5,51 \\
\text { puntos }\end{array}$ & $\begin{array}{c}5,39 \\
\text { puntos }\end{array}$ & $\begin{array}{c}5,73 \\
\text { puntos }\end{array}$ & $\begin{array}{c}5,5 \\
\text { puntos }\end{array}$ & $\begin{array}{c}5,15 \\
\text { puntos }\end{array}$ & $\begin{array}{c}4,92 \\
\text { puntos }\end{array}$ & $\begin{array}{c}5,9 \\
\text { puntos }\end{array}$ & $\begin{array}{c}4,7 \\
\text { puntos }\end{array}$ \\
\hline & $\begin{array}{l}\text { Que haya instrucciones claras } \\
\text { y simples sobre la HM a la } \\
\text { vista de todos los profesionales } \\
\text { sanitarios }\end{array}$ & $\begin{array}{c}5,21 \\
\text { puntos }\end{array}$ & $\begin{array}{c}5,17 \\
\text { puntos }\end{array}$ & $\begin{array}{c}5,36 \\
\text { puntos }\end{array}$ & $\begin{array}{c}4,5 \\
\text { puntos }\end{array}$ & $\begin{array}{l}5,06 \\
\text { puntos }\end{array}$ & $\begin{array}{c}4,5 \\
\text { puntos }\end{array}$ & $\begin{array}{c}5,8 \\
\text { puntos }\end{array}$ & $\begin{array}{c}5 \\
\text { puntos }\end{array}$ \\
\hline & $\begin{array}{l}\text { Que los profesionales sanitarios } \\
\text { reciban regularmente } \\
\text { retroalimentación sobre cómo } \\
\text { realizan la HM. }\end{array}$ & $\begin{array}{c}5,24 \\
\text { puntos }\end{array}$ & $\begin{array}{c}5,26 \\
\text { puntos }\end{array}$ & $\begin{array}{c}5,27 \\
\text { puntos }\end{array}$ & $\begin{array}{c}4,5 \\
\text { puntos }\end{array}$ & $\begin{array}{c}4,93 \\
\text { puntos }\end{array}$ & $\begin{array}{c}4,75 \\
\text { puntos }\end{array}$ & $\begin{array}{c}5,7 \\
\text { puntos }\end{array}$ & $\begin{array}{c}4,4 \\
\text { puntos }\end{array}$ \\
\hline & $\begin{array}{l}\text { Que usted realice } \\
\text { perfectamente la HM (siendo } \\
\text { un buen ejemplo para sus } \\
\text { colegas). }\end{array}$ & 5 puntos & $\begin{array}{c}5 \\
\text { puntos }\end{array}$ & $\begin{array}{c}5,09 \\
\text { puntos }\end{array}$ & $\begin{array}{c}4,5 \\
\text { puntos }\end{array}$ & $\begin{array}{c}5,03 \\
\text { puntos }\end{array}$ & $\begin{array}{c}4,67 \\
\text { puntos }\end{array}$ & $\begin{array}{c}5,5 \\
\text { puntos }\end{array}$ & 5 puntos \\
\hline & $\begin{array}{l}\text { Que se invite a los pacientes } \\
\text { a recordar a los profesionales } \\
\text { sanitarios que deben realizar } \\
\text { una buena HM. }\end{array}$ & $\begin{array}{c}3,52 \\
\text { puntos }\end{array}$ & $\begin{array}{c}3,55 \\
\text { puntos }\end{array}$ & $\begin{array}{c}3,67 \\
\text { puntos }\end{array}$ & $\begin{array}{c}4,5 \\
\text { puntos }\end{array}$ & $\begin{array}{l}2,96 \\
\text { puntos }\end{array}$ & $\begin{array}{c}2,67 \\
\text { puntos }\end{array}$ & $\begin{array}{c}2,6 \\
\text { puntos }\end{array}$ & $\begin{array}{c}3,78 \\
\text { puntos }\end{array}$ \\
\hline \multicolumn{2}{|c|}{$\begin{array}{l}\text { ¿Qué importancia concede su jefe/a de } \\
\text { Servicio médico (o la supervisora de } \\
\text { enfermería de su unidad) a que usted realice } \\
\text { una correcta HM? }\end{array}$} & $\begin{array}{c}5,21 \\
\text { puntos }\end{array}$ & $\begin{array}{c}5,22 \\
\text { puntos }\end{array}$ & $\begin{array}{c}5,09 \\
\text { puntos }\end{array}$ & $\begin{array}{c}5,5 \\
\text { puntos }\end{array}$ & $\begin{array}{l}5,16 \\
\text { puntos }\end{array}$ & $\begin{array}{c}5,08 \\
\text { puntos }\end{array}$ & $\begin{array}{c}5,6 \\
\text { puntos }\end{array}$ & $\begin{array}{c}4,78 \\
\text { puntos }\end{array}$ \\
\hline \multicolumn{2}{|c|}{$\begin{array}{l}\text { ¿Qué importancia conceden sus colegas a } \\
\text { que usted realice una correcta HM? }\end{array}$} & $\begin{array}{c}4,29 \\
\text { puntos }\end{array}$ & $\begin{array}{l}4,26 \\
\text { puntos }\end{array}$ & $\begin{array}{l}4,55 \\
\text { puntos }\end{array}$ & $\begin{array}{c}4 \\
\text { puntos }\end{array}$ & $\begin{array}{c}4,32 \\
\text { puntos }\end{array}$ & $\begin{array}{c}4,58 \\
\text { puntos }\end{array}$ & $\begin{array}{c}4,40 \\
\text { puntos }\end{array}$ & $\begin{array}{c}3,89 \\
\text { puntos }\end{array}$ \\
\hline \multicolumn{2}{|c|}{$\begin{array}{l}\text { ¿Qué importancia conceden los pacientes a } \\
\text { que usted realice una correcta HM? }\end{array}$} & $\begin{array}{c}4,22 \\
\text { puntos* }\end{array}$ & $\begin{array}{c}4,3 \\
\text { puntos }\end{array}$ & $\begin{array}{c}4,2 \\
\text { puntos }\end{array}$ & $\begin{array}{c}4 \\
\text { puntos }\end{array}$ & $\begin{array}{l}3,19 \\
\text { puntos }\end{array}$ & $\begin{array}{l}3,83 \\
\text { puntos }\end{array}$ & $\begin{array}{c}2,4 \\
\text { puntos }\end{array}$ & $\begin{array}{l}3,22 \\
\text { puntos }\end{array}$ \\
\hline \multicolumn{2}{|c|}{$\begin{array}{l}\text { ¿Cuánto esfuerzo considera que necesita } \\
\text { usted para realizar una buena HM cuando } \\
\text { presta atención a los pacientes? }\end{array}$} & $\begin{array}{l}2,10 \\
\text { puntos }\end{array}$ & $\begin{array}{l}2,04 \\
\text { puntos }\end{array}$ & $\begin{array}{c}2,18 \\
\text { puntos }\end{array}$ & $\begin{array}{l}3,5 \\
\text { puntos }\end{array}$ & $\begin{array}{c}2,48 \\
\text { puntos }\end{array}$ & $\begin{array}{c}3 \\
\text { puntos }\end{array}$ & $\begin{array}{c}1,9 \\
\text { puntos }\end{array}$ & $\begin{array}{c}2,44 \\
\text { puntos }\end{array}$ \\
\hline
\end{tabular}




\section{Discusión}

Nuestros resultados muestran que a pesar de que los PS de Nefrología refieren tener mayor formación en HM que los de Traumatología, estos últimos utilizan casi en su totalidad preparados de base alcohólica de forma habitual mientras que entre el PS de Nefrología no llega al $75 \%$. Esto puede estar relacionado con que entre el PS de Nefrología se cree de forma mayoritaria que la fricción causa más sequedad que el lavado de manos, mientras que entre el PS de Traumatología lo piensa aproximadamente la mitad; y con que la mitad de los PS de Traumatología piensa que la fricción es más eficaz contra los gérmenes que el lavado de manos frente al $21,6 \%$ de Nefrología. El estudio de Sánchez Payá y cols $^{13}$ indica la irritación cutánea excesiva y la sequedad de la piel como problemas derivados de la desinfección higiénica de manos.

Entre el $80-90 \%$ de los PS de ambas unidades se indica que la HM tras la exposición a fluidos corporales previene la transmisión de microorganismos al profesional sanitario y alrededor del $70 \%$ en ambos grupos lo indica como medida de prevención de transmisión de microorganismos al paciente. Esta actitud de protegerse podría explicarse porque los PS perciben el riesgo potencial de infección una vez que han entrado en contacto con un paciente y no se perciben como una posible fuente de infección para el paciente ${ }^{14}$. Sin embargo, si existen diferencias estadísticamente significativas entre ambos grupos en cuanto a la estrategia de HM ante determinadas situaciones, como antes de poner una inyección y después de hacer la cama del paciente, probablemente derivadas del mayor uso de soluciones hidroalcohólicas en el grupo de Traumatología.

Respecto a las percepciones existe una alta concienciación sobre la importancia de las IRAS respecto al desenlace de la enfermedad del paciente entre el PS de Nefrología en comparación con el de Traumatología, pudiendo estar relacionado con una formación mayor en el grupo de Nefrología. A pesar de estas diferencias en la formación, un mayor porcentaje de PS de Traumatología indicaron los 5 momentos para la HM indicados por la OMS (antes del contacto directo con el paciente, antes de realizar una tarea limpia o aséptica, después de exposición a fluidos corporales, después del contacto con el paciente y después del contacto con el entorno del paciente). La formación tiene un impacto positivo en cuanto a la adhesión al lavado de manos, como han demostrado distintos estudios ${ }^{15,16}$. Ambos grupos han mostrado una percepción positiva hacia la formación y retroalimentación sobre la $\mathrm{HM}$, por lo que acciones destinadas a la reducación en este aspecto serían bien acogidas y mejorarían tanto los conocimientos como las percepciones. La diferencia en cuanto a la puntuación a la pregunta de cuanta importancia conceden los pacientes a la correcta HM de los PS puede deberse a que el grupo de Nefrología trabaja con paciente crónicos, que conocen y se informan mucho sobre el proceso de su enfermedad.

Nuestro estudio es de los pocos que además de evaluar conocimientos, evalúa las percepciones de los PS respecto a la $\mathrm{HM}$; y no sólo en un grupo determinado de $\mathrm{PS}^{17}$. El tipo de muestreo puede ser una limitación de nuestro estudio, ya que la muestra seleccionada podría no ser representativa de los PS de ambas unidades. Futuros estudios podrían correlacionar los conocimientos y percepciones sobre la $\mathrm{HM}$ y su cumplimiento mediante estudios observacionales de conductas.

\section{Conclusiones}

Según nuestros resultados, los PS de Nefrología, al igual que los de Traumatología, en general, tienen conocimientos y percepciones adecuados sobre la $\mathrm{HM}$. Existen diferencias entre ambos grupos en cuanto a la formación sobre la $\mathrm{HM}$, el uso de soluciones de base hidroalcohólica y las percepciones sobre éstas.

Recibido: 20 Noviembre 2013

Revisado: 26 Noviembre 2013

Modificado: 28 Noviembre 2013

Aceptado: 28 Noviembre 2013

\section{Bibliografía}

1. Sociedad Española de Medicina Preventiva, Salud Pública e Higiene. Estudio de prevalencia de las infecciones nosocomiales en España. EPINE 1990-2011: 22 años. [Consultado 10/01/2012] Disponible en: http://www.sempsph.com/sempsph/ attachments/378_9-epine_1990-2011.pdf.

2. Pittet $D$, Donalson L. "Una atención limpia es una atención segura", primera iniciativa mundial de la Alianza Mundial de la OMS para la seguridad del paciente. IFIC. 2006; 2:135-40. 
3. Sociedad Española de Nefrología. Organización Nacional de Trasplantes. Registro Español de Enfermos Renales. Informe de diálisis y trasplante 2010. [Consultado 18 Diciembre 2011] Disponible en: http://www.senefro.org/modules.php?name $=w$ ebstructure\&idwebstructure $=128$.

4. Pugh-Clarke K, Donlon S, McCann M. CE: continuing education article. Prevention of infection in patients with chronic kidney disease part 1: application of infection control principles to the renal care environment. J Ren Care. 2010; 36(4):191-8.

5. Martín-Madrazo C, Salinero-Fort MÁ, CañadaDorado A, Carrillo-De Santa-Pau E, Soto-Díaz S, Abánades-Herranz JC. Evaluación del cumplimiento de higiene de las manos en un área de atención primaria de Madrid. Enferm Infecc Microbiol Clin. $2011 ; 29(1): 32-5$.

6. Novoa AM, Pi-Sunyer T, Sala M, Molins E, Castells $X$. Evaluation of hand hygiene adherence in a tertiary hospital. Am J Infect Control. 2007 Dec;35(10):676-83.

7. Naderi H, Sheybani F, Mostafavi I, Khosravi N. Compliance with hand hygiene and glove change in a general hospital, Mashhad, Iran: An observational study. Am J Infect Control. 2012 Mar 21. [Epub ahead of print].

8. Felembam 0, John WS, Shaban RZ. Hand hygiene practices of home visiting community nurses: perceptions, compliance, techniques, and contextual factors of practice using the World Health Organization's "five moments for hand hygiene". Home Healthc Nurse. 2012;30(3):152-60.

9. Chen $Y C$, Sheng WH, Wang JT, Chang SC, Lin HC, Tien $\mathrm{KL}$ et al. Effectiveness and limitations of hand hygiene promotion on decreasing healthcare-associated infections. PLoS One. 2011;6(11):e27163.

10. Erasmus $V$, Huis $A$, Oenema $A$, van Empelen $P, B o o g$ $M C$, van Beeck EH et al. The ACCOMPLISH study.
A cluster randomised trial on the cost-effectiveness of a multicomponent intervention to improve hand hygiene compliance and reduce healthcare associated infections. BMC Public Health. 2011;11:721.

11. Organización Mundial de la Salud. Cuestionario acerca de los conocimientos sobre la higiene de las manos destinado a los profesionales sanitarios. [Consultado 4 Noviembre 2011] Disponible en: http://www.who.int/gpsc/5may/tools/es/.

12. Organización Mundial de la Salud. Cuestionario de percepción sobre la higiene de las manos destinado a los profesionales sanitarios. [Consultado 4 Noviembre 2011] Disponible en: http://www.who. int/gpsc/5may/tools/es/.

13. Sánchez Payá J, González A, Galicia MD, García C, Garrigos I, Fuster M. Estudio piloto de ensayo clínico para evaluar la aparición de sequedad de la piel tras la utilización de soluciones alcohólicas. Medicina Preventiva 2004; X: 23-26.

14. Jang JH, Wu S, Kirzner D, Moore C, Youssef G, Tong $A$, et al. Focus group study of hand hygiene practice among healthcare workers in a teaching hospital in Toronto, Canada. Infect Control Hosp Epidemiol. 2010;31:144-50.

15. Tenías JM, Mayordomo C, Benavent ML, San Félix Micó M, García Esparza MA, Antonio Oriola R. Impacto de una intervención educativa para promover el lavado de manos y el uso racional de guantes en un hospital comarcal. Rev Calidad Asistencial. 2009;24(1):36-41.

16. Dierssen-Sotos $T$, Robles-García $M$, ValbuenaMolya S. Lavado de manos: experiencias de dos comunidades autónomas. Med Clin Monogr (Barc). 2008;131(Supl 3):60-3.

17. González-Cabrera J, et al. Cuestionario para evaluar en médicos conductas, conocimientos y actitudes sobre la higiene de manos. Gac Sanit. 2012. doi:10.1016/j.gaceta.2011.10.017. 